\title{
El misterio latinoamericano
}

\author{
Ricardo Yocelevzky Retamal \\ Universidad Autónoma Metropolitana, Unidad Cuajimalpa, Ciudad de México, México \\ Email.rretamal@correo.cua.uam.mx
}

\section{Óscar Cuéllar Saavedra}

Universidad Autónoma Metropolitana, Unidad Azcapotzalco, Ciudad de México.

México Email: oscarcuellar00@prodigy.net.mx

\begin{abstract}
Resumen: América Latina es un concepto, una construcción intelectual que intenta dar cuenta de un grupo de estados nacionales, variable en su composición pero con un núcleo estable, las ex colonias españolas y portuguesas del continente americano. Hoy no hay en América Latina un programa de desarrollo nacional alternativo a la integración al mundo capitalista global. Las experiencias nacionales que la izquierda dirige no constituyen una imagen como la que representó Cuba en los años sesenta, ni desde el punto de vista de los programas ni desde el liderazgo. Lo que se construyó en las décadas de los cincuentas y sesentas fue una conciencia de la relevancia de un proyecto continental que merecía ser respaldado por una teorización del conjunto, la construcción de un objeto de conocimiento al mismo tiempo que un actor político colectivo. Como quiera que sea, esta conciencia se desarrolló alrededor de las instituciones internacionales y nacionales residentes en Santiago de Chile y comenzó a desvanecerse junto con el proyecto de Allende y la Unidad Popular.
\end{abstract}

Palabras clave: América latina, Desarrollo, Instituciones internacionales.

\section{The Latin American mystery}

\begin{abstract}
Latin America is a concept, an intellectual construct that attempts to account for a group of national states, different in their composition but with a stable core, the former Spanish and Portuguese colonies on the american continent. Today there is no alternative national development program in Latin America but the global capitalist integration to the world. National experiences conducted by leftist governements do not constitute an image as the one Cuba represented in the sixties, neither from itrs programs nos from their leadership. What was built in the decades of the fifties and sixties was an awareness of the importance of a continental project that deserved to be supported by a theorization of its totality, the elaboration of an object of knowledge and at the same time a collective political actor. Either way, this awareness was developed around the international insitutions and national residents in Santiago de Chile and began to fade along with the project of Allende and the Unidad Popular.
\end{abstract}

Key words: Latin America, Development, International instituciones.

\section{O mistério da América Latina}

Resumo: A América Latina é um conceito, uma construção intelectual que tenta dar conta de um grupo de Estados-nação, variável na composição, mas com 
um núcleo estável, o ex-colônias Espanholas e Portuguêsas no continente americano. Hoje nao há um programa na América Latina de desenvolvimento nacional alternativo à integração global do capitalismo no mundo. Experiências nacionais que são dirigidas por a esquerda não constituem uma imagem como ela representou Cuba na década de sessenta, nem do ponto de vista de programas ou de liderança. $\mathrm{O}$ que foi construído nos anos cinquenta e sessenta, era uma consciência da importância de um projeto continental que merecia ser apoiado por uma teorização do conjunto, a construção de um objeto de conhecimento e ao mesmo tempo um ator político coletivo. De qualquer forma, essa consciência desenvolvida em torno das instituições nacionales e internacional residentes em Santiago de Chile começou a desvanecer-se, juntamente com o projeto de Allende e da Unidade Popular.

Palavras-chave: América Latina, Instituições Internacionales, Desenvolvimento.

\section{Introducción}

América Latina es un concepto, una construcción intelectual que intenta dar cuenta de un grupo de estados nacionales, variable en su composición pero con un núcleo estable, las ex colonias españolas y portuguesas del continente americano. ${ }^{1}$ A partir de eso, se agregan o restan estados o porciones de estados nacionales vecinos como las ex colonias británicas o francesas, particularmente islas del Caribe, donde por razones históricas, y a pesar de sus diferencias, Haití casi siempre, desde su independencia temprana con respecto al resto, ha estado vinculado y es considerado parte del núcleo latinoamericano. Lo central de este asunto es que la definición que se proponga de América latina, en distintos ámbitos de conocimiento, implica un programa, un proyecto, para estos países. Quizás por estar entre los primeros, la autoridad de su promotor y el grado de concreción que logró, el proyecto de Bolívar es una referencia casi obligada y hoy es una consigna. ${ }^{2}$

Hay siempre una dualidad en el objeto, al tratarse de un conjunto de estados nación. A veces se trata de un país que se propone como ejemplo y vanguardia y otras de una propuesta para el conjunto que presupone, como condición, algún tipo de unidad y/o concertación aunque sea parcial.

Otra constatación de hecho, no muy alentadora, la constituye la propuesta de Javier Pinedo de que, sea como sea, todos estos proyectos, al menos hasta ahora, han fracasado. Entre los proyectos que menciona Pinedo figuran propuestas intelectuales originadas en América Latina y de consecuencias que irían más allá del país del proponente principal. Por supuesto el primero es el proyecto de Bolívar, seguido por el romanticismo liberal. Después las ideas asociadas con Sarmiento y aquellas de Mariátegui. En la segunda mitad del siglo pasado se ubica el proyecto de la CEPAL y el intento representado por el gobierno de Allende en Chile entre 1970 y $1973 .^{3}$

La dicotomía que incorpora como uno de sus elementos a América Latina como alternativa a un agrupamiento de "las Américas" tiene su ori- 
gen en la política de los Estados Unidos de Norteamérica desde el siglo XIX, cuando el énfasis en la "latinidad" de la otra América implicaba una orientación europea, sobre todo en lo referente a los aspectos culturales.

La idea de una América Latina parece haber sido estimulada en Francia desde antes de la mitad del siglo XIX por quienes impulsaban los intereses imperialistas franceses como reemplazantes de la decadente metrópolis ibérica y en competencia con los “sajones", ingleses y norteamericanos. ${ }^{4}$ Sin embargo, quien parece haber utilizado el término "latinoamericano” por primera vez es Francisco Bilbao, según Miguel Rojas Mix, citado por Abramson, ${ }^{5}$ en una conferencia a la que se refieren los autores citados, pronunciada en Paris el 22 de junio de 1856, en la cual Bilbao denunciaba los imperialismos simétricos de Rusia y Estados Unidos.

Desde fines del siglo XIX, la denominación del conjunto de las excolonias españolas de América implicaba definiciones acerca del futuro posible, a partir de asumir la pertenencia “cultural” a un movimiento histórico que determinaba la dirección del progreso. La composición racial, mestiza, de la población representaba, para unos, la fuente de pertenencia a una corriente con esas aspiraciones y, para otros, un obstáculo a superar o, incluso, insuperable. Un ejemplo de la primera visión es el pensamiento de Francisco G. Cosmes, que en los noventas del siglo antepasado, hace más de cien años, escribía: “...el pueblo mexicano: una raza latina, en parte por la sangre, pero completamente latina por el espíritu, esto es, por la civilización, las costumbres, el carácter, los ideales, el modo de ser moral, la manera propia de concebir la existencia, la religión, el idioma, el idioma sobre todo, esa alma de las nacionalidades, una raza latina, repetimos, colocada en América como centinela avanzado frente al sajonismo desbordante tiene el deber imperioso de templar su fe y la conciencia de sus altos deberes en la verdadera fuente de donde proviene su existencia, de cobrar bebiendo en sus aguas, el vigor necesario para no desmayar en la terrible lucha con que continuamente se la amenaza, y de la cual está obligada a sacar incólume y con honra su personalidad independiente de todo yugo, lo mismo material que moral. ¿ Y habríamos para cumplir esta misión altísima de inspirarnos, los representantes actuales de esa raza, en otro espíritu distinto del que nuestra madre la España nos infundió, habríamos de buscar fuerzas y aliento en esa fuente exhausta casi de nuestro dudoso abolengo indígena, que, además de raquitismo cerebral y de la barbarie, representa la abyección de una servidumbre incurable, nacida desde tiempo inmemorial, bajo el yugo de la teocracia idólatra y del cacicazgo, y que los principios de igualdad del Evangelio, y la influencia de la civilización española, y la misma Independencia no han logrado redimir." 6

Después del quinto centenario, y con el surgimiento de movimientos sociales y políticos reivindicatorios de los pueblos originarios, la idea de una América Latina no busca negar su componente indígena sino incorporarlo en la definición de su situación histórica caracterizada como "colonialidad". 7 Sin embargo, esto significa que los movimientos de los pueblos originarios sean aceptados sin objeción. ${ }^{8}$ 


\section{Visiones actuales de la región}

Hoy se escribe sobre América Latina suponiendo que el lector comparte una noción de cierto sentido común acerca del objeto del discurso, o bien el autor se enfrenta casi inmediatamente con la opción de generalizar teóricamente o referirse a casos particulares como ejemplos. Es el caso del libro del editor de la sección "The Americas" de la revista "The Economist", Michael Reid, titulado "Forgotten Continent. The Battle for Latin America's Soul”, publicado en 2007. En el primer capítulo, el autor aventura un diagnóstico que es revelador de su visión de su objeto y, al mismo tiempo, ilustrativo de los problemas que ofrece el intento de hacer un juicio suficientemente general acerca de este conjunto de países: “...algunos países latinoamericanos están todavía tratando de consolidar el estado-nación una tarea decimonónica en Europa y en la Norteamérica anglófona.” 9

Vale la pena comenzar diciendo que es claro que para la revista “The Economist” América Latina como tal no constituye mas que un lugar geográfico puesto que la información referente a los países de la región se publica dentro de la denominación más incluyente "The Americas", que excluye a Estados unidos pero incluye a Canadá y a los países del Caribe anglófono. Ésta es una posición que es compartida en el mundo académico, donde los estudios de área también tienden a reducir, diluir o eliminar los estudios latinoamericanos. ${ }^{10}$

La atención que merece la región para esta revista se revela en dos características, una obvia, la portada, y otra más idiosincrática, los obituarios. En un poco más de cinco años, de febrero de 2005 a junio de 2010, el semanario ha dedicado seis portadas a temas latinoamericanos: uno a América Latina como tal, uno "la herencia de Fidel Castro", uno al despegue de Brasil, uno a Lula y Chávez, uno a Lula y uno a Chávez. En cuanto a los obituarios, una sección en que se destaca el fallecimiento más notable de la semana, los criterios son más amplios que para la portada, que obligadamente implica actualidad. En esta sección se incluyen artistas, criminales, intelectuales y hasta un loro y un pez. De los más de 250 obituarios del período, nueve estuvieron dedicados a personalidades latinoamericanas, incluyendo a Alfredo Stroessner y Augusto Pinochet.

De la afirmación citada más arriba dos elementos son importantes: primero, la intención parece ser el llamar la atención hacia un continente que va perdiendo importancia a los ojos de los países desarrollados, público para el cual se edita la revista en que se desempeña el autor, y, segundo, ésta, que puede parecer una descripción adecuada de América Latina desde muchos puntos de vista, tiene aspectos discutibles si se la mira con cuidado.

Estos aparecen, no tanto respecto de los países latinoamericanos como de lo que esa afirmación supone respecto de los estados-nación en Europa y Norteamérica. Al terminar el siglo XIX, en América latina había más estados nacionales organizados formalmente que en Europa. Se puede 
discutir el grado de integración nacional en los países latinoamericanos pero, hasta la primera guerra mundial, la forma política predominante en Europa eran los imperios, de cuya disolución como resultado de dicha guerra salieron los estados nacionales del siglo XX. Por otra parte, los procesos de unificación nacional de Alemania e Italia ocurrieron cuando ya las repúblicas latinoamericanas habían alcanzado, en general, la organización y distribución del territorio continental que conservan hasta hoy. Si para algunos países latinoamericanos todavía sus fronteras fueron motivo de conflicto, lo mismo se puede decir de las fronteras de los países europeos.

Una muestra de lo que se dice aquí es la conformación de la Sociedad de las Naciones (o Liga de las Naciones), antecedente de la Organización de las Naciones Unidas establecida luego de la Segunda Guerra Mundial, la cual fue establecida por el Tratado de Versailles, que fijó los términos de la paz al fin de la Primera Guerra Mundial. Al momento de su creación, en 1920, la Sociedad de las Naciones contaba con cuarenta países miembros fundadores. De éstos, 16 eran repúblicas latinoamericanas políticamente independientes. Un solo país africano, Liberia, cinco dominios británicos, 14 países europeos, de los cuales al menos dos debían su existencia a la guerra recién terminada (Checoeslovaquia y el reino de Yugoeslavia). Entre 1920 y 1930 se incorporaron a la Sociedad de las Naciones 11 países europeos, dos latinoamericanos y un africano y entre 1930 y 1940 la Sociedad de las Naciones se incrementó con dos países latinoamericanos, un africano dos europeos y un asiático. De este modo, en sus veinte años de existencia, la Liga de las naciones tuvo un total de 60 miembros de los cuales 20 fueron repúblicas latinoamericanas.

Estos hechos deberían relativizar, al menos, la rotunda afirmación acerca de atraso histórico de América Latina con respecto a Europa en la formación de estados nacionales independientes ${ }^{11}$. Sin embargo, la conciencia del "atraso" relativo de estos países surgida alrededor del centenario del comienzo de la independencia de España se transformó en otro tipo de discusión a partir de la segunda posguerra.

A mediados del siglo pasado, Kalman Silbert realizó un análisis de los progresos de los países latinoamericanos en la dirección de su tránsito a estados nacionales modernos. Primero justificó el enfocar a la región como un conjunto para luego establecer tipologías de diferenciación. Es interesante ver sus argumentos hoy: "Uno de los hechos que justifican un enfoque general de América Latina es el de que la mayoría de sus habitantes perciben una mancomunidad emocional. Han concretado esa conciencia en principios jurídicos internacionales de carácter único, en una organización regional, en su actitud dentro de los organismos internacionales, en el tratamiento preferencial para con sus vecinos y en sus teorías constitucionales." 12 A lo que parece referirse el autor citado es a la vigencia del proyecto industrializador representado por la Comisión Económica Para América Latina (CEPAL), al cual nos referiremos en detalle más adelante, por una parte, a pesar de que su foco de análisis es la modernización política de los países latinoamericanos. Por otra par- 
te, ya en ese tiempo Silvert hacía notar que en América Latina se habían producido más de 200 constituciones. ${ }^{13}$

Es muy importante examinar lo que ocurrió a partir de la fundación de las Naciones Unidas en la América Latina. Lo que surgió fue un proyecto de constitución de un actor colectivo en la política internacional a partir de un programa más o menos común de desarrollo nacional. Hoy se puede decir, como lo afirma Javier Pinedo, que este fue uno más de los proyectos frustrados de construir América Latina. Sin embargo, el examen de esa experiencia es importante para entender, al menos en parte, lo que ocurre hoy en este conjunto de países. La frustración o fracaso de este proyecto está en el origen de la situación actual de América Latina, por lo que deberá ser examinado con detalle. Sin embargo, se debe comenzar precisando las características de la situación prevaleciente hoy para rastrear su génesis en las experiencias pasadas.

Hoy no hay en América Latina un programa de desarrollo nacional alternativo a la integración al mundo capitalista global. Las experiencias nacionales que la izquierda dirige en Centro y Sudamérica (Argentina, Bolivia, Brasil, Ecuador, El Salvador, Nicaragua y Venezuela) no constituyen una imagen como la que representó Cuba en los años sesenta, ni desde el punto de vista de los programas ni desde el liderazgo. Esto puede ser visto como un efecto de la influencia de los medios de comunicación y su labor de construcción y proyección de imagen (o de asesinato de imagen, aunque algunos líderes parecen empeñarse en intentos de suicidio de imagen). Basta recordar que Salvador Allende nunca se distanció de Fidel Castro, a pesar de lo obvio de las diferencias de personalidad y de circunstancias, y que la imagen del paredón en Cuba fue usada con profusión en las campañas del terror de la derecha chilena en 1964 y en 1970, y ver que hoy, quien quiera parecer respetable, no puede darse el lujo de tomarse una foto con Hugo Chávez. Esto no es sólo el efecto alienante de los medios sino una derrota ideológica mucho más profunda y que requiere un tipo de análisis que ni los actores políticos ni los intelectuales otrora progresistas parecen estar dispuestos a enfrentar. Por el momento, lo único observable parece ser el transfuguismo y los cambios de posición individuales.

A pesar de todo, crisis económicas y financieras actuales, el catecismo que se recita hoy es el llamado “Consenso de Washington”. Éste es una codificación de un conjunto de medidas de política económica que representan casi exactamente lo contrario de lo que fue el proyecto común de desarrollo adoptado por la mayoría de los países latinoamericanos en la segunda posguerra. El siguiente resumen fue elaborado por quien codificó este decálogo en 1989 en un "paper” que buscaba fijar un esquema común para la exposición de los casos nacionales en una conferencia convocada por el Instituto de Economía Internacional de Washington D.C. (del cual es miembro el autor de dicho paper). ${ }^{14}$

“Mi trabajo (1990) afirmaba que había un amplio acuerdo en Was- 
hington de que las siguientes diez medidas de política económica eran deseables en casi todos los países latinoamericanos:

1. Déficit presupuestario...debería ser suficientemente pequeño como para ser financiado sin recurrir al impuesto que representa la inflación.

2. El gasto público debería ser redirigido desde áreas políticamente sensibles que reciben más recursos que los que puede justificar su contribución (rendimiento) económico... hacia campos descuidados pero de altos rendimiento económico y potencial para el mejoramiento de la distribución del ingreso, tales como la educación primaria y salud y la infraestructura.

3. Reforma tributaria...para ampliar la base impositiva y disminuir las tasas de impuestos marginales.

4. Liberalización financiera, apuntando a un objetivo último de determinación de las tasas de interés por el mercado.

5. Una tasa de cambio única, a un nivel suficientemente competitivo como para inducir un crecimiento rápido de las exportaciones no tradicionales.

6. Reemplazo rápido de las restricciones cuantitativas al comercio por tarifas que sean progresivamente reducidas hasta alcanzar una tasa uniformemente baja, en el orden del10 al 20 por ciento.

7. Abolición de las barreras a la inversión extranjera directa.

8. Privatización de las empresas estatales.

9. Abolición de las regulaciones que impiden la entrada de nuevas firmas o restringen la competencia.

10. Provisión de seguridad al derecho de propiedad, especialmente al sector informal.”

Que estas medidas signifiquen revertir las políticas desarrollistas que por décadas se habían aplicado en distintas versiones en América Latina es el resultado de confrontaciones políticas e ideológicas que aparentemente no se relacionan directamente con ellas. Esto se debe a que los campos de confrontación ideológicas se mantuvieron voluntaria o involuntariamente separados. Es por esto que es necesario revisar una historia supuestamente muy conocida. En ella hay que consignar y distinguir los campos de las ideas en ciencias sociales, en política y las aplicaciones por distintos gobiernos de los programas que se derivan o pueden ser emparentados con estas ideas. 


\section{La América latina desarrollista}

Se puede decir que la América Latina de las políticas desarrollistas, desde los cincuenta hasta los setentas, tiene autor y domicilio conocido. Sin embargo, no parece ser del conocimiento común el que los inicios fueron inciertos y conllevaron enfrentamientos ideológicos y político - burocráticos dentro del naciente orden internacional de la posguerra, estructurado a través del sistema de las Naciones Unidas. Es dentro de ese marco que surgió el centro de pensamiento latinoamericano constituido por la Comisión Económica Para América Latina (CEPAL) y su principal animador y líder intelectual, Raúl Prebisch. La importancia de Prebisch en esta organización y en la influencia que ella alcanzó es tal que una parte del origen de la CEPAL está documentado en la detallada biografía de éste realizada por Edgar J. Dosman. ${ }^{15}$

Este biógrafo de Prebisch ubica el nacimiento de esta visión y este programa para América Latina en la presentación que éste hizo en La Habana en la segunda sesión de CEPAL en mayo de 1949. Lo que Prebisch expuso es el conocido artículo "El desarrollo económico de la América Latina y algunos de sus principales problemas”. ${ }^{16}$

Puede parecer anecdótico hoy, pero los detalles de la producción de este documento son ilustrativos del clima en que fue formulado: la Comisión Económica Para América Latina tenía un mandato de tres años, que es lo más que aceptó la representación de Estados Unidos en el Consejo Económico y Social de las Naciones Unidas (ECOSOC) para que la región recibiera la atención que buscaba frente a la preferencia que se daba a la reconstrucción de Europa y el lejano oriente (para lo cual se habían formado las primeras comisiones económicas, para Europa, con sede en Ginebra y la comisión para Asia y el Lejano Oriente, con sede en Bangkok). La propuesta de creación de una comisión para América Latina fue presentada en el ECOSOC por el gobierno de Chile, con el apoyo de Cuba, Perú y Venezuela, encontrando la oposición de los Estados Unidos de Norteamérica, Gran Bretaña, Canadá y otros países industrializados, así como la Unión Soviética. ${ }^{17}$

Estados Unidos prefería apoyar a la Unión Panamericana, relanzada como Organización de Estados Americanos en abril de 1948 en la Novena Conferencia Interamericana en Bogotá, sólo dos meses antes de la reunión fundacional de CEPAL, programada para junio de 1948 en Santiago de Chile. Esta situación es sólo una de muchas manifestaciones de la falta de inocencia de la diferencia conceptual entre "las Américas” y América Latina”. La conferencia de Bogotá estuvo marcada por el "bogotazo”, un evento de significación nacional, probablemente único, pero que fue tomado como muestra de un cierto clima político latinoamericano que despertó temores acerca de una probable repetición en otros países de la región.

Todo este contexto, del cual sólo se da aquí un brochazo, contribuyó a que la intervención de Prebisch en La Habana se convirtiera en un hecho 
político más allá del contenido económico del documento. Hay que decir que esta exposición es la primera expresión de lo que llegaría a ser conocido como la "tesis Prebisch-Singer", pero que en este caso se convirtió en un manifiesto latinoamericano.

Más tarde el nombre de Prebisch será asociado con una (de sólo dos) contribuciones originales a las ciencias sociales en el siglo XX en que figure un latinoamericano según el recuento hecho por Karl Deutch en los años setentas del siglo pasado. ${ }^{18}$

El que Santiago de Chile se convirtiera en la sede de esta idea de América Latina no parece haber sido el designio de nadie. Sin embargo, puede haber relación entre el hecho que la propuesta de una comisión económica para la región haya sido promovida por el gobierno chileno y que la reunión de fundación haya sido convocada en Santiago. Hay más en esta historia que casualidades. En la reestructuración del sistema internacional al final de la Segunda Guerra Mundial aparecían oportunidades de acción y de constitución de bloques de países. Según DSosman, el biógrafo de Prebisch ya citado, Chile intentaba establecer su liderazgo entre las delegaciones latinoamericanas en los organismos internacionales. ${ }^{19}$

Luego que el éxito del primer informe contribuyó a que el mandato se prorrogara, Santiago ofrecía varias ventajas, no la menor en esa época era estar tan lejos de Washington como fuera posible. Nadie podría considerar a Prebisch un simpatizante comunista, la Unión Soviética también se había opuesto a la idea de una comisión regional al comienzo, y sin embargo, en una carta en 1990, Hans Singer recuerda la atmósfera de esos días "Prebisch, como latinoamericano y a salvo, lejos, en Santiago, era mucho menos vulnerable que yo que estaba en Nueva York durante el período de McCarthy, y la tesis Prebisch-Singer era considerada subversiva” ${ }^{20}$

El que la CEPAL fijara su sede en Santiago significó que, debido a un acuerdo de la Asamblea General, otras agencias de las Naciones Unidas debieran instalarse allí también. ${ }^{21} \mathrm{La}$ CEPAL, como institución con un programa de desarrollo nacional atractivo para el conjunto de los países latinoamericanos y con un líder intelectual, (Prebisch había sido elegido su secretario ejecutivo en 1950 y en 1951, al término del primer mandato de tres años se resolvió la permanencia de la comisión) resultó un imán para la generación de economistas jóvenes que en la década de los cincuenta construyeron una idea nueva de América Latina, a través del conocimiento mutuo y el intercambio de experiencias. ${ }^{22}$ En palabras de un economista joven de la CEPAL en la época: "recuerdo que nos quedábamos discutiendo de política con los amigos latinoamericanos, había venezolanos, centroamericanos, mexicanos, (recuerdo a Noyola) hasta las once de la noche, cuando prácticamente nos tenían que echar de las oficinas". "No recuerdo otro período tan arrebatador en el trabajo como el de esos años.”23

En la constitución de esta conciencia latinoamericana hubo una tendencia a evitar definiciones políticas demasiado específicas o coyuntura- 
les. En esto no era menor la situación del mismo Prebisch con respecto al gobierno de Perón en Argentina y, al mismo tiempo, la política de Argentina con respecto a Naciones Unidas. Sin embargo, la orientación a la realización práctica de las ideas que animaban la elaboración de diagnósticos y planes de desarrollo para los diferentes países exigía la ampliación de la perspectiva desde dos ángulos: por un lado la incorporación del contexto histórico-social que redefinía el sentido de los planteamientos económicos en cada situación nacional y, por otro, la necesidad de formación de personal capacitado para la compilación e interpretación de información acerca de la economía y la sociedad. Estos estímulos reforzaron el movimiento hacia la masificación de la educación superior en los países, donde fueron apareciendo las escuelas que formaban personal especializado en las "carreras nuevas” (principalmente de las ciencias sociales).

Para reforzar estas tendencias aparecieron organismos internacionales que tuvieron mayor o menos significación y durabilidad en el escenario de la producción de conocimiento e información acerca de América Latina. Un caso importante es la Facultad Latinoamericana de Ciencias Sociales (FLACSO) creada en $1957 .{ }^{24}$

Todo este complejo de circunstancias condujo a que Santiago de Chile se transformara en el terreno privilegiado del transcurso regular de las elaboraciones ideológicas y del desarrollo de unas ciencias sociales con orientación latinoamericana.

En los años cincuenta se concentró el interés en la elaboración de informes anuales cada vez más completos del estado de las economías nacionales de América Latina. Al mismo tiempo, las ideas de la CEPAL eran difundidas entre los políticos jóvenes sin distinción de partidos o tendencias ideológicas. Del mismo modo se podía encontrar políticos identificados con las ideas de la socialdemocracia que con la democracia cristiana que adoptaban estas ideas y proponían para sus países programas de desarrollo económico inspirados en ellas. ${ }^{25}$ Los economistas jóvenes elaboraron ene esos años diagnósticos que fueron ampliados reinterpretaciones de las historias económicas nacionales, ${ }^{26}$ todo lo cual constituyó un horizonte ideológico en el que América latina, como actor colectivo en el plano internacional, desempeñaba un papel protagónico.

La coyuntura de finales de los cincuenta resultó en cambios inesperados en panorama político-ideológico de la región. Hoy se recuerda como punto de quiebre la huida de Fulgencio Batista de La Habana el 1 de enero de 1959 y el comienzo de la revolución cubana. Pero hay que recordar que esta última es un caso especial dentro de una categoría más amplia. Entre 1957 (derrocamiento de Rojas Pinilla en Colombia) y 1961 (asesinato de Trujillo en República Dominicana) también había caído Pérez Jiménez en Venezuela en 1958 y, en Chile, Salvador Allende, en su segunda postulación, había perdido la elección presidencial por escasa diferencia de votos frente a Jorge Alessandri. E ese marco, la caída de Batista, y su reemplazo por Fidel Castro, podría haber sido un episodio más y no el fin de una época 
para la política de los Estados Unidos de Norteamérica hacia América Latina. La coyuntura que termina por definirse con el asesinato de Trujillo y la Segunda Declaración de La Habana, ${ }^{27}$ marca la cancelación de aquella época en que para Estados Unidos no importaba que gobernara un "hijo de puta con tal que fuera 'su' hijo de puta”. ${ }^{28}$

La política hacia América Latina de la administración Kennedy se basó en el aislamiento de Cuba y la prevención de cualquier posibilidad de repetición de la revolución en otro país de América Latina. Este objetivo incluía acciones militares, políticas y de planeación. En las primeras estuvo la reformulación de las defensas de los países de la región en función de la hipótesis de un "enemigo interno", en las segundas estuvo el lanzamiento de un programa de ayuda al desarrollo llamado “Alianza para el Progreso" y el tercero, que afectó particularmente a las ciencias sociales latinoamericanas por algún tiempo, fue el programa de investigación conocido como "Plan Cámelot”.

El primer objetivo se logró en 1961, en la Conferencia de Punta del Este, Uruguay, en la cual la OEA expulsó a Cuba de la organización. Sin embargo, la ruptura de relaciones diplomáticas con Cuba por parte de los gobiernos latinoamericanos no fue automática. Algunos países, como Chile tardaron hasta el siguiente año para hacerlo y México nunca rompió sus relaciones con Cuba. Sobre el entrenamiento en contrainsurgencia de oficiales de las fuerzas armadas de los países latinoamericanos en las escuelas de Estados Unidos en Panamá o en su mismo territorio hay abundante documentación.

Sin embargo, desde el punto de vista ideológico, lo más complejo fue la Alianza Para el Progreso. Aparentemente esta implicaba que, al fin, el gobierno de los Estados Unidos de Norteamérica entendía los planteamientos acerca del desarrollo económico de los países de la región y adoptaba como orientación de su política exterior hacia ellos las necesidades que se derivaban de esos programas. Así lo relatan Levinson y De Onís en su examen de la alianza extraviada.

Por otra parte, Dosman afirma que de diez puntos principales que componían el discurso de John Kennedyen el que se presentaba oficialmente la Alianza Para el Progreso, ocho estaban incluidos en un memorándum enviado por los jefes de la OEA, el Banco Interamericano de Desarrollo y la CEPAL, el cual había sido redactado en borrador, manuscrito, por Raúl Prebisch incluyendo hasta una cita textual del memorándum redactado por Prebisch. ${ }^{29}$

La redefinición de las relaciones entre Estados Unidos y América Latina significó un realineamiento de los intelectuales latinoamericanos con respecto a la revolución cubana y un realineamiento de los organismos internacionales en relación a Estados Unidos y entre ellos mismos.

La década de los sesenta estuvo marcada por los efectos de la revolución cubana. Por una parte, el modelo de acción política que había con- 
ducido a Fidel Castro al poder, la guerra de guerrillas, fue imitado, con algunas variaciones en toda América Latina (con la excepción de Costa Rica).Por otra, la ideología de la revolución cubana tuvo efectos muy variados. Si la segunda Declaración de La Habana era una proclamación de la lucha por la independencia nacional de los países latinoamericanos, en el contexto de la Guerra Fría, las fuerzas anticomunistas reaccionaron fuertemente contra Cuba en tanto quienes sacaban conclusiones de la experiencia cubana constituían un arco ideológico mucho más amplio que el restringido a los partidos comunistas.

Santiago de Chile fue el escenario privilegiado de una buena parte de las disputas ideológicas de esa década. A la presencia de organismos internacionales y escuelas asociadas para la formación de personal en la producción y aplicaciones de conocimientos de ciencias sociales, como la planificación económica y social, se agregaron factores políticos tales como el que el gobierno chileno de 1964 a 1970 fuera escogido como vitrina para mostrar las posibilidades de una alternativa democrática a la revolución cubana, fuertemente apoyada por la Alianza Para el Progreso. ${ }^{30}$ A esto se agregaron los golpes de estado en Brasil (1964) y Argentina (1966) que llevaron a Santiago a un número importante de científicos sociales, profesores y estudiantes, que contribuyeron significativamente a la producción del pensamiento latinoamericano de la época.

El arco ideológico es amplio y, además, no se desarrolla en un solo plano. Tomando como eje las propuestas básicas de la CEPAL, un desarrollo nacional que tenga como eje la industrialización, aparecen las críticas que asumen la forma de teorías alternativas que, primero explicarían el fracaso o incluso la inviabilidad de dichas propuestas y, segundo, justificarían un programa alternativo construido, principalmente, a partir de la experiencia de Cuba. En el plano político se desarrolla lo que se llamó el "castrismo" y en el plano de las ciencias sociales las varias versiones de la teoría de la dependencia o, como prefieren algunos, no sin razones, las teorías de la dependencia.

América Latina es construida ahora como el campo en que el futuro es de reforma o revolución. El espectro político aparece desplazado hacia una izquierda en la que hasta la política de Estados Unidos parece concurrir. Lo que todos estos enfoques tienen en común es que teorizan "una" América Latina, construida como proyecto común o como experiencia común, en la que se distinguen tipos o situaciones, estructurales o históricas, pero no se duda de la legitimidad del objeto regional. Hay una conciencia de pertenencia y, en algunos casos un "nacionalismo" latinoamericano que busca modelos y alternativas en las experiencias nacionales.

No se puede atribuir directamente a la revolución cubana la polarización y la radicalización de la lucha política que condujo a la victoria de Allende y la izquierda en Chile en 1970. El clima ideológico, como se ha descrito, era más complejo. Para lo que interesa aquí, la proyección de la experiencia de la Unidad Popular era no sólo una vía alternativa para Amé- 
rica Latina (cuando ya la imitación y búsqueda de reproducción de modelos de cambio era un programa y no sólo una eventualidad posible). Como afirma Pinedo, la propuesta de Allende en Chile era una idea de América Latina, que ya estaba de alguna manera en la conciencia de muchos latinoamericanos que habían participado en la construcción de las varías propuestas que tenían como destinatario a los países latinoamericanos considerados como parte de una entidad y un proceso conjunto.

La derrota del proyecto de Allende adquirió una proyección desmesurada si se toma a Chile como país y su participación en América Latina sin considerar el escenario ideológico que venía constituyendo desde hacía más de una década. Para el movimiento comunista europeo, Chile pasó a ser un caso de discusión en el contexto de lo que se llamó el eurocomunismo. En otros casos, el impacto que provocó la brutalidad de la dictadura de Pinochet, como castigo al intento chileno de búsqueda de un camino de desarrollo nacional que apuntara al socialismo como meta por vías políticas constitucionales fue equiparable, en la solidaridad que despertó, al impacto que generó la guerra civil española casi cuarenta años antes. Poco después se vio la consecuencia en el mundo ideológico de la aplicación de una política económica inspirada en las visiones más radicales de la economía neoclásica, en la visión monetarista de Milton Friedman, quien asesoró al gobierno de la dictadura, y en la ocupación de los mandos de la economía chilena por un grupo sólidamente formado en la ideología liberal que llegó a ser conocido como los "Chicago boys" debido a su trayectoria como economistas formados en la Pontificia Universidad Católica de Chile y más tarde, en virtud de un convenio existente por décadas, en la Universidad de Chicago, donde sus mentores principales fueron el ya mencionado Friedman y Arnold Harberger, ambos vinculados como asesores a la dictadura militar.

Lo que sigue es historia muy conocida: el ascenso de la ideología laxamente llamada neoliberal y su generalización en todos los países de América Latina, especialmente luego de la crisis de la deuda externa de los años ochenta. El triunfo de la ideología liberal en el campo de la economía tuvo un rápido desarrollo y ya a comienzos de los noventas se anunciaba que "10,000 hombres de Harvard, una nueva generación de tecnócratas entrenados en los Estados Unidos está reconfigurando las economías de América Latina”. ${ }^{31}$ Esta vez los mentores mencionados son Rudiger Dornbusch del MIT y Jeffrey Sachs de la universidad de Harvard.

En el mismo reportaje se cita a Harberger disociando la acción económica de los "excesos" de la dictadura: "Nunca enseñamos ideología, a pesar de lo que alguna gente dice", "no es justo". ${ }^{32}$ Sin considerar los problemas de justicia que puedan estar involucrados en todo esto,el reemplazo de una visión por otra puede ser visto como los resultados sucesivos de enfrentamientos ideológicos en el campo de la ciencia económica. Entre la “alguna gente” a la que alude Harberger está sin duda André Gunder Frank, quien en los primeros años de la dictadura chilena dirigió una carta abierta a Friedman y Harberger recordando los años de Frank en la universidad de Chicago y cómo su primer acercamiento a Chile ocurrió en compañía del segundo. ${ }^{33}$ 
Jeffrey Sachs escribe sobre el "valor social de la moderación, la confianza, el respeto por la experticia y la búsqueda de la verdad en la gestión pública”. Su visita a Chile se produjo por una invitaciónd de su "estimado ex compañero de curso, el presidente Sebastián Piñera, y de un ex estudiante estrella y coautor, Felipe Larraín.” ${ }^{34}$

La visión de la economía detrás del “Consenso de Washington” ya citado tiene una diferencia central con las concepciones de la CEPAL y las teorías de la dependencia que consiste en construir las economías nacionales como unidad de análisis que sólo pueden ser agrupadas por relaciones entre ellas o por semejanzas empíricas. No hay lugar para la construcción de un actor colectivo en la arena internacional, o no por lo menos como algo relevante desde el punto de vista de la economía. Por el contrario, lo que se construyó en las décadas de los cincuentas y sesentas fue una conciencia de la relevancia de un proyecto continental que merecía ser respaldado por una teorización del conjunto, la construcción de un objeto de conocimiento al mismo tiempo que un actor político colectivo, con diferencias respecto de esto entre las variantes que surgieron tanto en el campo político como en el de las ciencias sociales. Como quiera que sea, esta conciencia se desarrolló alrededor de las instituciones internacionales y nacionales residentes en Santiago de Chile y comenzó a desvanecerse junto con el proyecto de Allende y la Unidad Popular. 


\section{Notas}

${ }^{1}$ Carlos M. Rama hace una exploración de las posibilidades de fundamentar el concepto desde distintos puntos de vista disciplinarios en “¿Existe América Latina?”, capítulo 1 de Sociología de América Latina, pp.9-23.

${ }^{2}$ El cambio de nombre de Venezuela (a República Bolivariana de Venezuela) efectuado bajo el gobierno de Hugo Chávez contrasta con el título del libro de Jorge Volpi "El insomnio de Bolívar”. Sin embargo, son prueba de lo que se afirma aquí.

${ }^{3}$ Esta cita puede no reflejar fielmente y en detalle el pensamiento del autor citado pues provienen de su exposición oral en el 51 Congreso de Americanistas, México, D. F., 2009.

${ }^{4}$ Abramson, Pierre-Luc: Las utopías sociales en América Latina en el siglo XIX, pp. 52-53.

${ }^{5}$ Op. cit., p110.

${ }^{6}$ Cosmes, Francisco G.: La dominación española y patria mexicana, pp. 42-43.

${ }^{7}$ Quijano, Anibal: “El 'movimiento indígena’ y las cuestiones pendientes en América Latina”. En Argumentos, N50, enero-abril, 2006, pp.51-77.

${ }^{8}$ Ver, por ejemplo, las declaraciones de Sergio Villalobos, historiador chileno, Premio Nacional de Historia 1992, al periódico Las Últimas Noticias, el 10 de octubre de 2010.

${ }^{9}$ Op. cit. P.9.

10 Dunkerley, James, "Dreaming of Freedom in the Americas:Four Minds and a Name." Institute for the Study of the Americas. Inaugural Lecture, 25 October 2004.

${ }^{11}$ En la introducción al primer volumen de su obra The Modern World System, Immanuel Wallerstein menciona la importancia de esta paradoja latinoamericana para definir una teoría del desarrollo.

${ }^{12}$ Silvert, Kalman H.: La sociedad problema. Reacción y revolución en América Latina, p. 31.

${ }^{13}$ Op. cit., p.37.

${ }^{14}$ Williamson, John: “The strange history of the Washington consensus”, en Journal of Post Keynesian Economics, Vol.27, N², Winter 2004-5, pp. 195-206.

${ }^{15}$ Dosman, Edgar J.: The Life and Times of Raúl Prebisch. 1901-1986.

16 “Escrito en 1949 como introducción al Estudio económico de la América Latina 1948. Posteriormente fue publicado en el Boletín económico de la América Latina, Vol. VII, núm. 1, febrero de 1962.” Nota al pié en la publicación del mismo documento en Adolfo Gurrieri (compilador), La obra de Prebisch en la cepal, p. 99.

${ }^{17}$ Dosman, Op. Cit., p. 236. 
Polis, Revista de la Universidad Bolivariana, Volumen 10, $N^{\circ}$ 29, 2011

18 “Innovaciones básicas en las ciencias sociales, 1900-1965”, en Ciencia y Desarrollo, Año VII, N³9, julio-agosto de 1981, CONACYT, México, pp, 33-36.

${ }^{19}$ Op. Cit. P.236.

${ }^{20}$ Dosman, Op. Cit., p. 522.

${ }^{21}$ Franco, Rolando: La FLACSO clásica (1957-1973), p.34.

22 "Santiago became a movement - almost a church - under the firm guidance of the 'great heretic””. Dosman, Op. Cit., p. 274.

${ }^{23}$ Entrevista con Jacobo Schatan, Santiago de Chile abril de 2009.

${ }^{24}$ Franco, Op. Cit.

${ }^{25}$ Por ejemplo algunos de los escritos de Eduardo Frei Montalva en esos años.

${ }^{26}$ Entre estas destacan las obras de Aldo Ferrer, Celso Furtado y Aníbal Pinto.

${ }^{27} 4$ de febrero de 1962.

${ }^{28}$ Fórmula cínica con que Franklin Délano Roosevelt definió la política de Estados Unidos hacia Latinoamérica al recibir en Washington a “Tacho” Somoza, dictador de Nicaragua.

${ }^{29}$ Dosman Op. Cit., p.358.

${ }^{30}$ Yocelevzky, Ricardo: La Democracia Cristiana Chilena y el gobierno de Eduardo Frei. 1964-1970.

${ }^{31}$ Revista Newsweek, 10 de diciembre de 1990, pp.20-23.

${ }^{32}$ Loc cit., p. 22.

${ }^{33}$ Frank, André Gunder: "First Open Letter to Arnold Harberger and Milton Friedman, August 6, 1974 (Hiroshima Day)” en Economic Genocide in Chile. Monetarist Theory Versus Humanity.

${ }^{34}$ Sachs, Jeffrey: “Las lecciones de Chile en liderazgo.” Herald Tribune, 22 de octubre de 2010, traducido y publicado por La Nación, Santiago de Chile, sábado 24 de octubre de 2010.

Recibido: 28.05.2011

Aceptado: 06.07.2011 